\title{
Perfumes naturales en la elección de pareja
}

\author{
Carlos Daniel Juárez-Santiago
}

\section{Resumen}

La teoría de la selección sexual propuesta por Darwin intenta explicar la evolución de ciertos rasgos que son importantes en la elección de pareja. Los animales se comunican mediante señales visuales, auditivas, químicas o táctiles. La comunicación química es una de las más utilizadas a nivel intra e interespecífico y se caracteriza por emplear una serie de aromas o perfumes naturales (feromonas) durante la elección de pareja. Los perfumes naturales presentan diferentes estructuras químicas, dependiendo de factores como función, medio en el que se emplean, y duración y nivel de especificidad de los grupos taxonómicos que los utilizan. El entorno en el que se desenvuelven los organismos es importante ya que trae consigo variaciones en la producción de feromonas y esto está relacionado con la salud del individuo.

En este artículo se abordará el uso de los perfumes naturales en la elección de parejas en diferentes animales y cómo estos son empleados en el proceso de reproducción.

Palabras clave: comunicación química, selección sexual, feromonas, perfumes, elección de pareja.

\section{NATURAL PERfumes IN CHOOSING A PARTNER}

\begin{abstract}
The theory of sexual selection proposed by Darwin tries to explain the evolution of certain traits that are important for mate choice. Animals communicate through visual, auditory, chemical or tactile signals. Chemical communication is one of the most employed at an intra and interspecific level and consists of using a series of aromas or natural perfumes (pheromones) during couple's choice. Natural perfumes have diverse chemical structures depending of factors like function, medium in which are employed, and duration and level of specificity in the taxonomic groups that use them. The environment in which the organisms developed is important because it implies variations in pheromone production, which is related to individual health.

This article describes perfumes' use during mate choice in different animals and how they are employed during the reproduction process.
\end{abstract}

Keywords: chemical communication, sexual selection, pheromones, perfumes, mate choice. 


\section{Carlos Daniel Juárez-Santiago}

vdharius@gmail.com

Biólogo egresado de la Universidad Del Mar campus Puerto Escondido, Oaxaca, México. Con experiencia en monitoreo de fauna silvestre y conducta animal, así como investigación de campo. Temas de interés: fauna silvestre, biología de la conservación y ecología de la conducta.

\section{Introducción}

La teoría de la selección sexual postula que algunos rasgos que presentan individuos del mismo sexo son resultado de la competencia por el acceso a la cópula y de la elección del otro sexo, que generalmente considera factores como la inteligencia, confiabilidad, salud, posibilidad de aportar recursos y características físicas de los individuos para proporcionar ventajas relacionadas con la reproducción (Hernández y Cerda, 2012; Darwin, 1888). La teoría hace distinción entre dos tipos de caracteres sexuales: los primarios, como los órganos genitales, que se relacionan directamente con la función reproductora; y los caracteres sexuales secundarios, que aparentemente no están conectados directamente con la reproducción, pero que en conjunto participan en el éxito o fracaso reproductivo. Dentro de éstos, el uso de sustancias químicas es considerado un mecanismo importante para la elección de pareja (Cordero y Santolamazza, 2009).

La comunicación química es el principal medio para establecer relaciones entre los individuos, mediante el intercambio de sustancias que se pueden oler o saborear. Aunque es difícil distinguir entre el olfato y el gusto como sentidos independientes, hablaremos de un sentido químico general, que cumple funciones de orientación, obtención de alimento y agua, y reconocimiento de amigos, enemigos y parejas (Juárez, 1987). Los perfumes naturales o feromonas son señales químicas emitidas por un individuo, capaces de alterar el comportamiento o la fisiología de otro. Cumplen con la función de ser mensajeros en la interacción de dos o más individuos, por lo que es importante conocer sus características, su funcionalidad como atrayentes de pareja, los costos biológicos que implica su producción, y los conflictos que conlleva su uso, con el fin de entender cómo se comunican los animales y cómo la comunicación química juega un papel importante en el momento de elegir pareja (Gutiérrez y Contreras, 2002).

\section{¿En qué consisten los perfumes?}

Los perfumes naturales son considerados como una forma de lenguaje dentro de las especies, basado en moléculas que pueden ser traducidas por los sentidos químicos (Juárez, 2017). Dichas moléculas son relativamente grandes, compuestas entre 10 y 17 átomos de carbono y con un peso molecular que varía de 180 a 300 daltones. Los compuestos de este tamaño o mayor logran 
alta especificidad y gran potencia, sin embargo, la síntesis de moléculas grandes y complejas es más costosa, por lo que su producción tiene que ser cuidadosamente regulada por el organismo, sobre todo considerando que el tamaño de las feromonas está relacionado con la volatilidad y por lo tanto la difusibilidad, la cual disminuye al aumentar el peso molecular.

Las feromonas pueden actuar como cebadoras, señaladoras, moduladoras o liberadoras. Para definirlas necesitan satisfacer ciertos criterios: tener efectos conductuales inmediatos y no atribuibles a otro estímulo sensorial, ser sustancias específicas, que exista poca o nula influencia de la experiencia en su funcionamiento, y que se sepa el estado reproductivo de quien las secreta. En la naturaleza los animales no actúan fuera de un contexto ambiental y rara vez secretan perfumes en ausencia de señales sensoriales. Es decir, para que la comunicación sea efectiva, se necesita que se desarrolle una serie de factores físicos, sociales y ambientales. En ratones se han identificado, a partir de la orina, las estructuras químicas de feromonas que sirven como atractores sexuales: pertenecen principalmente al grupo de las cetonas, irazinas, esteres, terpenos y tiazoles (Delgadillo, 2005; Gutiérrez y Contreras, 2002, ver imagen 1). Los dos tipos principales de feromonas en estas relaciones son las feromonas liberadoras que inician modelos de conducta específicos y sirven como poderosos atrayentes sexuales, marcan territorio, provocan una reacción de alarma o la agregación de los individuos; así como las feromonas iniciadoras o cebadoras, que disparan cambios metabólicos o fisiológicos, particularmente el sistema endocrino, que está asociado a la maduración sexual, el crecimiento o la metamorfosis (López, 2002).

Imagen 1. Los ratones son la especie más estudiada en cuanto al comportamiento en función de las feromonas (fuente: Wikimedia cammons).

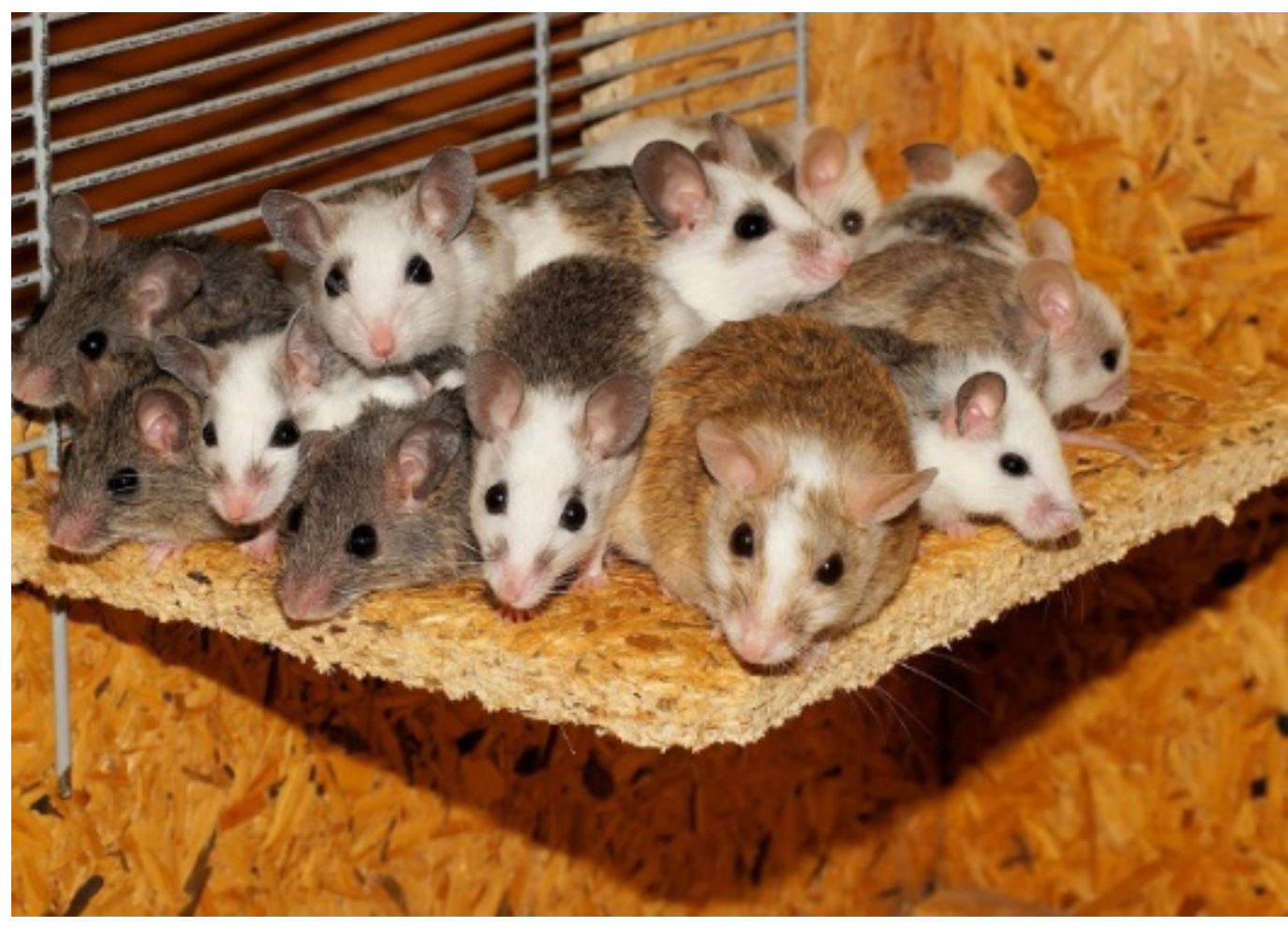




\section{Los perfumes naturales como atrayentes de pareja}

De acuerdo con Darwin -y el conocimiento actual concuerda con él-, la selección de parejas se da mediante dos mecanismos: la selección dentro del mismo sexo (intrasexual) y la que ocurre entre diferentes sexos (intersexual). La selección intrasexual funciona como una batalla entre individuos del mismo sexo. De manera general, aunque también ocurre de manera contraria y Darwin estaba consciente de ello, los machos luchan entre sí para tener acceso a la hembra; así, se han seleccionado ciertos rasgos como astas y cuernos que los ayudarían en dicha contienda. El segundo mecanismo, la selección intersexual, en la mayoría de los casos ocurre en las hembras, quienes tienen preferencia por determinado macho, aunque, una vez más, podría ocurrir de manera contraria, que el macho tenga preferencia por cierta hembra. De esta manera, dicho mecanismo ha contribuido a la selección de algunas características como la cola del pavo real, las señales químicas, la coloración de aves y peces, el canto de las aves y los rituales de cortejo, entre otros (Andersson, 1994).

Los perfumes naturales son relevantes pues permiten la comunicación entre miembros de una misma especie. Uno libera estas moléculas volátiles y otro las percibe a través de su sistema olfativo (Paredes Cordero et al., 2016). Y estas señales químicas son utilizadas como un medio para atraer pareja en una gran variedad de especies.

En insectos, tenemos el caso del escarabajo de harina (Tenebrio spp.) que produce olores brindando información del estado y salud de los individuos, lo que les da la posibilidad de elegir un compañero correcto (Pölkki et al., 2012). Asimismo, ciertos insectos presentan especificidad en los compuestos que producen, los cuales varían según la edad del adulto, el sexo y el estado de apareamiento, y participan como feromonas, llamadas kairominas, así como en el reconocimiento de género y especies en el caso de insectos sociales (Peralta Falcon, 2018). En las mariposas se han identificado feromonas sexuales que los machos usan para inducir a las hembras al apareamiento. Por ejemplo, algunas especies poseen un penacho de pelo muy fino y fragante en el extremo del abdomen, a la vista del vuelo de una hembra de la misma especie el macho se ubica sobre la cabeza de la hembra y abre sus finos pelos para desprender las feromonas, entonces la hembra se posa y rápidamente se aparea con el macho. La hormona responsable es la pirrolizidinona, la cual es biosintetizada a partir de los alcaloides que producen las plantas de las cuales se alimentan (Robles, 1994).

Un modelo empleado para el estudio de los efectos de las feromonas en la elección de parejas es el de la salamandra Plethodon jordani, debido al papel primordial en esta especie de la comunicación química en la elección de pareja. Se ha identificado que las feromonas involucradas son producidas por pequeñas glándulas localizadas debajo de la barbilla (Rollman et al., 2000). En los mamíferos, como el ratón, se han clasificado feromonas sexuales y para la reproducción, se han descrito varios de sus pormenores (Gutiérrez y Contreras, 2002, ver imagen 2). 
Imagen 2. Estructura química de algunos de los compuestos urinarios que poseen actividad química en el ratón. Se han identificado algunas cetonas como: a) 2-hexanona, 2-heptanona y 4-heptanona; b) ésteres como: n-pentil acetato; $\mathbf{c )}$ algunos terpenos como: E-E-afarneseno (fragmento tomado y modificado de GutierrezGarcia, 2002).<smiles>CCCCC(C)=O</smiles>

a<smiles>CCCCCC(C)=O</smiles><smiles>CCCC(=O)CCC</smiles>

b<smiles>CCCCCOC(C)=O</smiles>

c<smiles>C=CC(C)=CCC=C(C)CCC=C(C)C</smiles>

\section{Función de los perfumes en la elección de pareja}

El funcionamiento de los perfumes y su efectividad se basan en una correcta comunicación entre el emisor y el receptor, lo que vincula coevolutivamente a los órganos que emiten a los perfumes junto con los que reciben estas señales.

El desarrollo de glándulas endocrinas y diferenciación del sistema nervioso se

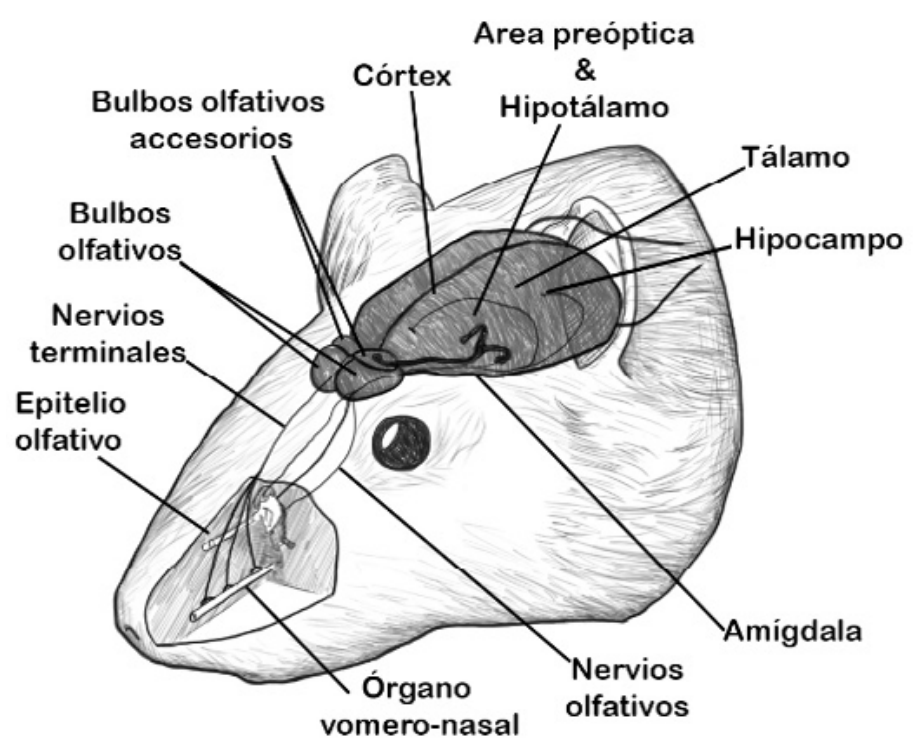

relaciona con el uso de los perfumes para funciones de señalización (Gutiérrez y Contreras, 2002; Juarez, 2017). En los mamíferos se identificaron dos sistemas quimiosensoriales independientes, que responden a estas señales químicas, capaces de traducir la información feromonal en el cerebro: el sistema olfativo principal y el sistema vomeronasal (Delgadillo, 2005, ver imagen 3). El sistema olfativo principal tiene como función el recibir olores, pero las feromonas son detectadas por el órgano vomeronasal, este órgano está ubicado en la cavidad nasal y tiene un segmento de mucosa olfatoria localizada a lo largo del tabique nasal (Guevara Guzmán, 2004).

Imagen 3. El sistema olfativo principal y el sistema vomeronasal del ratón (imagen elaborada por José Eduardo Hernández Cortes).
De igual forma las feromonas pueden tener influencia directa en las emociones, los estados de ánimo y los sentimientos tanto negativos como positivos. Las feromonas pueden incluso inducirlos, llegando a cambiar la percepción social de las personas, por una modificación en el procesamiento de la información en las áreas límbicas del cerebro. Por lo tanto, el uso de perfumes permite la manipulación directa de la cognición. Otras funciones de las señales 
químicas en los mamíferos son el reconocimiento específico de los individuos de la misma especie o camada, la delimitación del territorio e indicación del nivel jerárquico de dominancia. Además, su atractivo junto con el de otras características sexuales secundarias ha moldeado aspectos morfológicos y conductuales de los animales, por lo que es probable que la psicología animal, incluyendo la humana, haya evolucionado paralelamente a los estímulos sexuales para percibirlos, interpretarlos y responder a ellos adecuadamente (Grammer et al., 2003).

\section{¿Es costoso para los animales producir perfumes?}

La adaptación de las especies a diferentes ambientes es un factor que influye en los costos y los beneficios en la producción de cada señal, el gasto energético que requiere producirlas dependerá del receptor y del ambiente en el cual está el mensaje; la calidad y la cantidad emitida; y de las condiciones de cada individuo, los que tienen una buena salud toleran una mayor producción de feromonas.

En el caso de la polilla del racimo (Lobesia botrana) se identificó que la producción de feromonas en los machos responde a su condición actual: los machos de mayores tallas son los que producen señales con mayor efectividad, atrayendo una mayor cantidad de hembras, las cuales, se ha visto, tienden a invertir mayor energía en la producción de huevos cuando copulan con machos de mayores tallas (Harari et al., 2001). Los caracteres sexuales secundarios extravagantes son costosos, y al ser demasiado llamativos, en algunos casos, reducen las perspectivas de supervivencia y sólo se pueden mantener si son elegidos entre la población (Grammer et al., 2003).

\section{Los conflictos clásicos relacionados con el uso perfumes durante la elección de pareja}

Se han descrito algunos fenómenos relacionados con las feromonas en ratones. Por ejemplo, la orina del macho puede promover un reinicio de los ciclos estrales o reproductivos de una hembra, también llamados celo o calor (Rippe, 2009), así como la sincronización de ciclos cuando están en grupos, a este fenómeno se le conoce con el nombre de efecto Whitten. Incluso si este fenómeno no es costoso de manera directa, sí promueve la descendencia del macho dominante y que las hembras puedan realizar los cuidados parentales en grupo, lo que mejoraría el éxito de las camadas. También se da el efecto Vandenbergh, que consiste en el aceleramiento del inicio de la pubertad en hembras, inducido por la presencia del olor de la orina de un macho, lo que se traduce en una mayor probabilidad de dejar descendencia (Gutiérrez y Contreras, 2002).

Otro conflicto es el efecto Bruce, comúnmente conocido en ratones de laboratorio. Sucede cuando una hembra recién preñada encuentra a un macho distinto que con el que copuló. En este contexto es probable que la hembra sufra de una interrupción de la gestación promovida por la presencia de la orina 
Imagen 4. Dos hembras con sus crías de la especie Theropithecus gelada (imagen tomada de Wikimedia commons). de los machos. Este efecto se había identificado sólo en ratones y bajo condiciones de laboratorio; sin embargo, en condiciones naturales recientemente se observó a un grupo de primates (Theropithecus gelada) en los que de manera natural el macho tiene la capacidad de secretar sustancias que provocan la interrupción de la gestación, lo que garantizaría que las crías son del macho dominante en curso y promovería el cuidado parental de parte de éste (Wolff, 2003; Roberts et al., 2012; ver imagen 4).

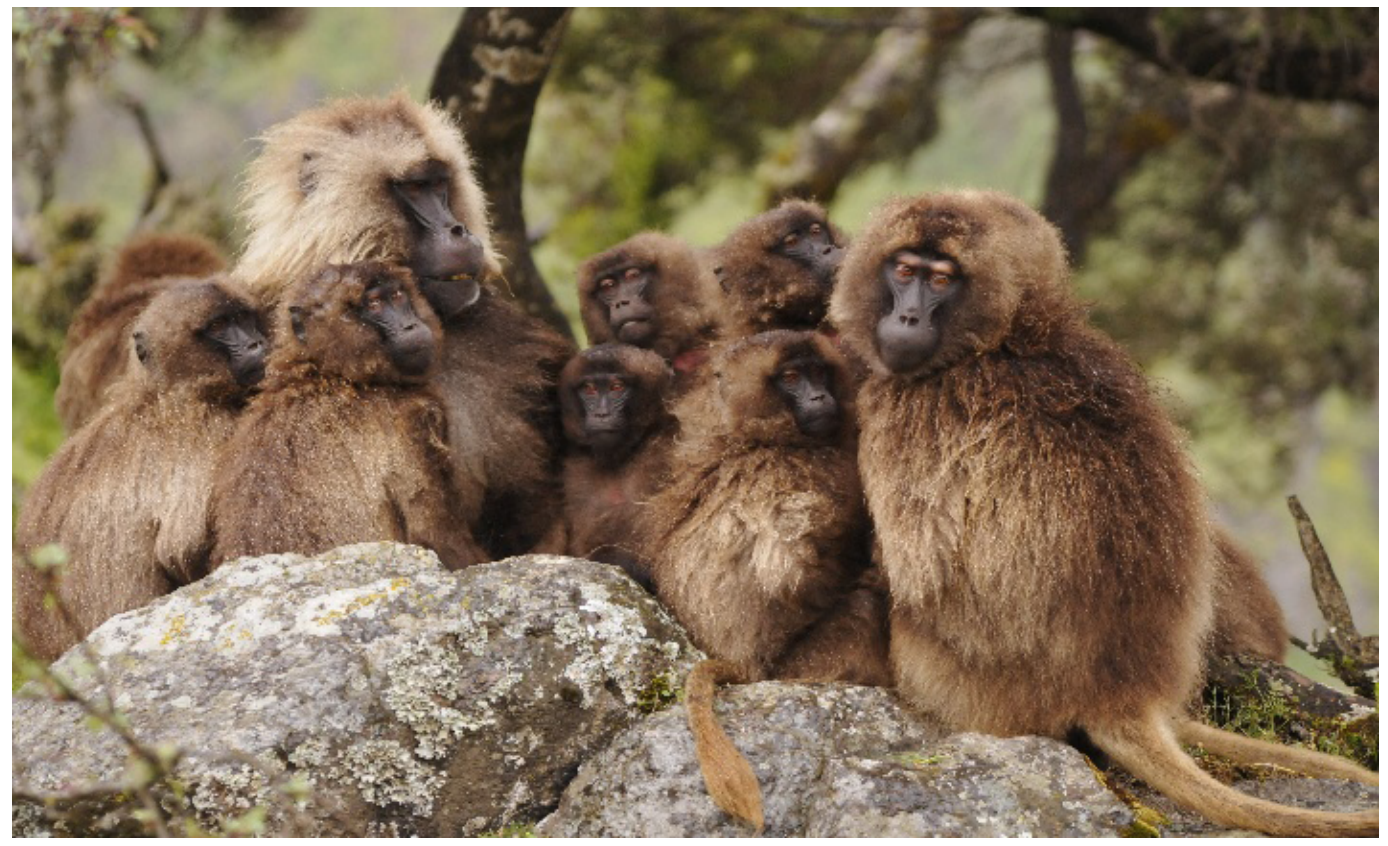

Otros conflictos que acarrean las señales químicas son la agresión y la territorialidad. Las conductas agresivas se presentan cuando los intereses de dos o más individuos entran en conflicto, y comúnmente se desencadenan cuando los individuos interactúan para establecer una jerarquía de dominancia, - para determinar la territorialidad. En los casos en los que se integra un nuevo individuo a un grupo, puede ser tomado como intruso debido a que las feromonas que libera no son familiares para los demás. En este caso, el nivel de conflicto que determinará la condición social y territorial dentro del grupo dependerá de cómo se interprete la información química y corporal (Gutiérrez y Contreras, 2002).

\section{Conclusión}

El empleo de perfumes naturales en el mundo animal es tan cotidiano como el uso de perfumes o lociones en la vida de cada persona; son utilizados como una herramienta al momento de elegir pareja, donde las características de cada individuo se verán reflejadas en los perfumes liberados. Esto ha promovido que en muchos organismos se seleccionen caracteres sexuales secundarios que garantizan su descendencia y la perpetuación de sus genes. 
Los perfumes en general cumplen funciones importantes en las interacciones entre individuos, tanto en el medio social como en la elección de pareja, ya que es una manera para identificar la salud de los organismos, aunque posteriormente su presencia puede promover una serie de conflictos como el efecto Bruce o Vandenbergh.

Las feromonas cumplen con la función de ser el mensajero en la interacción de dos o más individuos, por lo que es relevante seguir investigando sus características, su funcionalidad como atrayentes de pareja, los costos biológicos que implica su producción, y los conflictos que conlleva su uso; lo anterior con el fin de entender cómo se comunican los animales y cómo la comunicación química, en particular los perfumes naturales, juega un papel importante en el momento de elegir pareja.

En la actualidad no se ha identificado el valor fundamental de los perfumes en la elección de parejas en los seres humanos, por lo tanto, se requieren investigaciones adicionales para comprender mejor estas conductas. En cambio, los perfumes naturales en los animales representan un campo grande de estudio y el conocer e identificar cómo los organismos los utilizan es importante para desarrollar estrategias en la reproducción o su control.

\section{Referencias}

Andersson, M. B. (1994). Sexual selection. S. I.: Princeton University Press.

* Cordero Rivera, A. y Santolamazza Carbone, S. (2009). Darwin y la selección sexual después de la cópula. Revista Digital Universitaria, 10(6). URL: http://www.ru.tic.unam. mx/tic/bitstream/handle/123456789/1505/855.pdf? sequence=1\&isAllowed=y.

- Darwin, C. (1888). The descent of man and selection in relation to sex (vol. 1). Londres: Murray.

* Delgadillo, D. C. (2005). Feromonas. Lo que el viento se llevó. Revista Casa del Tiempo, 3. URL: www.uam.mx/difusion/revista/junio2005/02.pdf.

- Grammer, K., Fink, B., Møller, A. P. y Thornhill, R. (2003). Darwinian aesthetics: sexual selection and the biology of beauty. Biological Reviews, 78(3): 385-407. DOI: https://doi.org/10.1017/S1464793102006085.

* Guevara Guzmán, R. (2004) ¿Cuál es el papel de las feromonas en la conducta sexual humana? Revista de la Facultad de Medicina, 47(1): 16-20 URL: http://www. medigraphic.com/pdfs/facmed/un-2004/un041e.pdf.

* Gutiérrez-García, A. G. y Contreras, C. M. (2002). Algunos aspectos etológicos de la comunicación química en ratas y ratones de laboratorio. Revista Biomédica, 13(3): 189-209. URL: http://www.medigraphic.com/pdfs/revbio/bio-2002/bio023f.pdf.

* Harari, A. R., Zahavi, T. y Thiéry, D. (2011). Fitness cost of pheromone production in signaling female moths. Evolution, 65(6): 1572-1582. Dol: https://doi.org/\%20 10.1111/j.1558-5646.2011.01252.x. 
* Hernández-López, L. E. y Cerda-Molina, A. L. (2012). La selección sexual en los humanos. Salud mental, 35(5): 405-410. URL: http://www.scielo.org.mx/scielo. php? script=sci arttext\&pid=S0185-33252012000500007.

* Juárez Vea, J. (1987). Feromonas: olor y comunicación. Elementos. 12(2): 36-46. URL: http://www.elementos.buap.mx/num12/pdf/36.pdf.

* López, P. (2002). Comunicación intraespecífica en reptiles mediante señales químicas: la utilización de señales fiables para decidir comportamientos. Revista Española de Herpetología: 49-59. Revisado diciembre 2017. URL: www.herpetologica. org/revespherp/volespecial 2002/049-059\%20lopez.pdf.

- Paredes Guerrero, R., Fernández, A. A., Juárez, C., Tapia, S., Figueroa, Á., Criou, X. y Encinas, A. (2016). Los olores del amor. URL: http://www.unamiradaalaciencia.unam. $\mathrm{mx} / \mathrm{stc}$ metro/consulta stcm pdf.cfm? vArchivoStcm=123.

* Peralta Falcón, R. (2018) Hidrocarburos cuticulares que intervienen en el reconocimiento intraespecifico de toxotrypana curvicauda (diptera: tephritidae). URL: https://tesis.ipn. $\underline{m \times / h a n d l e / 123456789 / 25711 . ~}$

* Pölkki, M., Krams, I., Kangassalo, K. y Rantala, M. J. (2012). Inbreeding affects sexual signalling in males but not females of Tenebrio molitor. Biology letters, 8: 423-425. DOI: https://doi.org/10.1098/rsbl.2011.1135.

* Rippe, C. A. (2009). El ciclo estral. En Dairy Cattle Reproduction Conference (pp. 111116).

* Roberts, E. K., Lu, A., Bergman, T. J. y Beehner, J. C. (2012). A Bruce effect in wild geladas. Science, 335(6073), 1222-1225. Dol: https://doi.org/10.1126/ science.1213600.

* Robles Caycho,J. (1994). Comunicación química. Revista de Química, VIII (2): 163-175. URL: http://revistas.pucp.edu.pe/index.php/quimica/article/viewFile/5534/5530.

* Rollmann, S. M., Houck, L. D., y Feldhoff, R. C. (2000). Population variation in salamander courtship pheromones. Journal of Chemical Ecology, 26(12), 27132724. URL: http://people.oregonstate.edu/ houckl/pdfs/Rollmann\%20et\%20 al\%202000\%20JChemEcol.pdf.

Wolff, J. O. (2003). Laboratory studies with rodents: facts or artifacts? AlBS Bulletin, 53(4), 421-427.

\section{Cómo citar este artículo}

* Juárez Santiago, Carlos Daniel (2019). Perfumes naturales en la elección de pareja. Revista Digital Universitaria (RDU). Vol. 20, núm. 2 marzo-abril. Dol: http://doi. org/10.22201/codeic.16076079e.2019.v20n2.a2

RECEPCIÓN: 23/01/2018 APROBACIÓN: 12/02/2019 